\title{
POLICY The complexities of race and health
}

\author{
Authors: Adwoa Danso ${ }^{A^{*}}$ and Yaa Danso ${ }^{B *}$
}

It is widely accepted that race and related social factors largely underpin patients' access to healthcare, and even have a direct impact on patients' care. The reality that racism is the source of these health inequalities, and that racism within health organisations compounds the issue, undeniably means that racism is a public health issue. The ongoing pandemic has not only shone a light on underlying issues that have silently plagued the Black, Asian and minority ethnic community but has helped us understand the devastating impact of racism. Closing the gap in these populations is required to ensuring equitable access. This article aims to highlight how racism impacts people of colour within the healthcare system and beyond and calls for dismantling of the structural racism that exists within the NHS and other UK organisations.

KEYWORDS: racism, health inequality, ethnic minorities, medical racism, history of racism in medicine

DOI: $10.7861 /$ fhj.2020-0225

\section{Introduction}

It is widely accepted that race and related social factors largely underpin patients' access to healthcare, and even have a direct impact on the provision of care. ${ }^{1}$ Racism is an undeniable public health issue compounding these health inequalities at both an organisational and population level.

COVID-19, as of the start of 2021, has infected over 87 million people globally and has taken the lives of over 78,000 people in the UK. ${ }^{2}$ The ongoing pandemic has not only highlighted underlying issues that have silently plagued the Black, Asian and minority ethnic (BAME) community but has also helped us understand the devastating impact of racism. Closing the inequality gap in these populations is required to ensure equitable access.

The hesitancy to recognise racism as a growing issue does not help these matters. In this article we hope to highlight how racism impacts people of colour within the healthcare system and beyond, with the optimism that this may serve to unpack the structural racism that exists within the NHS and other UK organisations.

Authors: Asessional GP, Barking, Havering and Redbridge University Hospitals NHS Trust; ${ }^{B}$ gastroenterology specialist registrar, Pennine Acute NHS Trust, Bury, UK; * joint first authors.

\section{COVID-19 and racism}

Recent and current events have made clear that disparities in health outcomes are significantly affected by racism. The NHS workforce statistical data from January 2020 showed BAME healthcare workers make up $21 \%$ of the workforce but $63 \%$ of healthcare worker deaths from COVID-19. ${ }^{3}$ Initially there were a number of attempts to explain these worryingly disproportionate figures as caused by biological mechanisms, including suggestions that vitamin $D$ deficiency, specific comorbidities and genetics played a role. ${ }^{4,5}$ The idea that racism was a factor was initially dismissed. Only after a public inquiry were racism, discrimination, stigma and distrust identified as a fundamental factors affecting health, exposure risk and disease progression in COVID-19. ${ }^{6}$

Highlighting racism as a contributory factor in the deaths from COVID-19 was monumental. It allowed the public and government to look more critically at previous concerns. The 2020 report Black people, racism and human rights produced by the Joint Committee on Human Rights of the House of Commons and House of Lords has illustrated the unequal treatment of Black people across various institutions, including education, employment, healthcare and within the judicial system. The report also revealed numerous failures to implement previous recommendations within the NHS. The report recognised that the mortality of Black women during childbirth is five times higher compared to White women - 'The NHS acknowledges and regrets this disparity but has no target to end it. The Government must introduce a target to end the disparity in maternal mortality between Black and White women' - and that this should be implemented as a 'matter of urgency'?

\section{Ethnic minorities in the NHS}

In recent times, the NHS has been subject to accusations of institutional racism. For healthcare workers and the public, the subsequent findings were confirmation of what many had suspected. In 2018, 29\% of BAME healthcare staff experienced harassment, bullying or abuse from other NHS staff within the previous 12 months, up from $26 \%$ in 2016 . This increase was over double the increase seen with non-BAME staff: reports from nonBAME workers showed an increase of only $1.2 \%$ over the same period to $24.2 \%$. Meanwhile, $29.8 \%$ of BAME staff experienced bullying or abuse from patients or the public in the previous 12 months compared with $27.8 \%$ of White NHS staff in $2018 .{ }^{8}$ Fear of ramifications is also felt on an individual basis when speaking out against concerns. For members of staff, it may be difficult to express concerns because of trepidation that these would not be taken seriously. We have a culture where we are accustomed to not 'see race'. However, claiming not to see racism only serves 
to further delegitimise this lived experience of those facing this reality every day.

The Freedom to speak up report, an independent review, set out key principles and actions to encourage an open and honest reporting culture in the NHS. ${ }^{9}$ The report included the finding that BAME NHS staff who raised concerns were more likely to be victimised or ignored by management and co-workers compared to their White colleagues. It also examined how BAME doctors were more likely to be referred to the GMC and to receive tougher sanctions than White doctors. ${ }^{9}$ Within NHS trusts, $7.4 \%$ of executive board members were from a BAME background. This is abysmal when compared to $19.7 \%$ of the BAME workforce across all the NHS trusts. ${ }^{8}$ It is vital to have diversity among those in leadership roles to ensure that that issues of particular significance to marginalised groups are represented. Indeed, recruiting minorities into leadership roles has been shown to reduce the negative experience of minority staff. ${ }^{10}$ We can go further by implementing or adapting the 'Rooney Rule', first introduced in 2003 by the National Football League, which requires all teams to interview at least one individual from an ethnic minority background for head coaching positions. ${ }^{11}$

The Royal College of General Practitioners (RCGP) was put into the spotlight in 2013 after the British Medical Journal (BMJ) reported that BAME graduates trained in the UK were more likely to fail the final GP clinical skills assessment compared to White candidates. ${ }^{12}$ It was disappointing that the initial response from the RCGP was to threaten legal action against the $B M J$, despite the paper being peer-reviewed and accepted for publication. ${ }^{13}$ However, it was the RCGP who were then taken to court by the British Association of Physicians of Indian Origin (BAPIO). The RCGP have since stated they want to work with BAPIO to 'find the best way of supporting the small number of trainees who fail the CSA component of the MRCGP licensing exam, to give them every chance of passing.'.4

Two years prior to this, a meta-analysis of 23 reports comparing the academic performance of medical students and doctors found that those of non-White ethnicity underperform academically compared with their White counterparts. The results of candidates from different ethnic groups were reviewed from undergraduate assessments, postgraduate assessments, machine-marked written assessments and practical clinical assessments among others, with very similar findings. Specifically, of the 36 sets of data that were reviewed, 35 showed a negative effect of non-White ethnicity and only one showed no effect. None showed a positive effect of being non-White. ${ }^{15}$

Several years after these two studies were published, it remains apparent that even now much more is required to tackle the racial disparities within the medical profession. ${ }^{13}$ Racial bias is systemic; we must address this and be ready to tackle this issue head on without fear of ramification to ensure a fair and just method of training and of assessing current and future doctors.

\section{Bias and health outcomes}

The first principle within the NHS constitution is based on equality: 'The NHS provides a comprehensive service, available to all irrespective of gender, race, disability, age, sexual orientation, religion or belief. It has a duty to each and every individual that it serves. ${ }^{16}$ But despite these aspirations, racism and discrimination continue to exist within the NHS; indeed, racism is a social construct. ${ }^{17}$ It is clear from the science taught to healthcare professionals that there is very little difference in the biological make up between those of different ethnicities and there is certainly no credible research to suggest superiority. ${ }^{18}$ However, the historical baggage of stereotypes, implicit attitudes, thoughts and feelings frame the lens through which people of colour are defined and interacted with. As a result, healthcare within the NHS is not exempt from this racial bias and there is growing and emerging research to confirm this. An article from the USA reviewed beliefs associated with racial bias in the assessment of pain and treatment recommendation. It looked at false beliefs (eg 'Black people's skin is thicker than White people's skin', 'Black people's nerve-endings are less sensitive than White people's nerveendings' and 'Whites, on average, have larger brains than Blacks') and biological differences between races held by medical students, resident doctors and lay people. Staggeringly, 50\% reported that at least one of the false belief items was possibly, probably, or definitely true. The study found that half of those White medical students and residents in their sample held these beliefs and most worryingly, they showed a racial bias in their treatment, providing inadequate treatment recommendations $15 \%$ of the time. ${ }^{19}$

This evidence is not limited to one study; a review evaluating the treatment of appendicitis found that Black children were less likely than White children to receive appropriate analgesia within the emergency department. The review found the largest disparities within the groups with the highest pain scores, where $24.5 \%$ of Black patients compared with $58.3 \%$ of White patients received analgesia. ${ }^{20} \mathrm{~A}$ cohort study of 217 patients with long bone fractures found Black patients are less likely to receive emergency analgesia than similarly injured White patients; $74 \%$ versus $57 \%$ with Black individuals having a $66 \%$ greater chance of not receiving any analgesia. $^{21}$

A systematic review of 15 similar studies investigated the extent to which implicit racial/ethnic bias exists among healthcare professionals and examined the effect these had on healthcare outcomes. The general conclusion was that most healthcare providers appear to have implicit bias in terms of positive attitudes towards Whites and negative attitudes towards people of colour. Levels of implicit bias against Black, Hispanic and dark-skinned people were relatively similar. Some associations between implicit bias and healthcare outcomes were non-significant; however, results also showed that implicit bias was most significantly related to patient-provider interactions and health outcomes. ${ }^{22}$ This is particularly important because the patient-provider interaction is what dictates the effectiveness of a health system as a whole.

Correlational evidence indicates that biases are likely to influence diagnosis and treatment decisions. This impact was demonstrated in a US study which found Black newborn babies cared for by White doctors faced 430 more fatalities per 100,000 births than White newborns. There was no statistical difference in fatalities of White babies cared for by Black doctors. ${ }^{23}$ The data is alarming and may illustrate the effects of racial bias within healthcare.

Bias and false beliefs are built on stereotypes that stream into the networks within our society. These attitudes are often automatically activated and can influence a person's behaviour without conscious volition. The substandard outcomes mentioned above are not just seen within healthcare establishments but can also play a part in guidelines and local protocols. Clearly, this can have widespread and stark implications on healthcare outcomes within minority communities. 
In the UK the prevalence of hypertension is higher in ethnic minority groups. Significantly higher mean blood pressure levels are reported among both Afro-Caribbean and South Asian populations. ${ }^{24}$ The National Institute for Health and Care Excellence (NICE) hypertension guideline states to offer a calcium-channel blocker (CCB) to adults of African-Caribbean origin (of any age) who do not have Type 2 Diabetes as step 1 of antihypertensive treatment. ${ }^{25}$ There are a number of issues with the evidence used to formulate this guidance, which affects $18 \%$ of the UK Black population. ${ }^{26}$ The guidance is based on the US study ALLHAT. The 2002 study looked at how the incidence of non-fatal myocardial infarction and deaths related to coronary heart disease varied depending on antihypertensive treatments. ${ }^{27}$ The usefulness of the study is questionable; it was limited to individuals over the age of 55 and there was no control for socioeconomic status and access to healthcare. There are limitations of the use of race within clinical tools. If race-based tools are to be utilised they must be cautiously and appropriately incorporated. ${ }^{28}$ There is also growing evidence that links chronic psychological stress to cardiovascular disease. ${ }^{29}$ Exposure to racism has been shown to increase the risk of hypertension. ${ }^{30}$ There is a growing need for further research aligning to the needs of BAME populations rather than extrapolating limited data. Racialisation of clinical guidelines together with the existence of racial bias unquestionably impacts patient health.

Creating guidelines that are a fit for all is challenging. We also recognise there is still some way to go in creating more diverse cohorts within research. There has been a lack of inclusion of minority groups within medical research, seemingly due to low demand for evidence..$^{31}$ The publication of Beyond the data: Understanding the impact of COVID-19 on BAME groups has emphasised the gaps that exist within data collection specifically pertaining to ethnicity and the need for this to be rectified. ${ }^{6}$ There is value in allocating funding to research areas conducted by BAME colleagues to truly explore and understand the complexities of health inequalities. ${ }^{32}$ There are recommendations of the development and the use of methodical and analytical procedures to allow subpopulations to be meaningfully included in research. ${ }^{33}$ Nevertheless, BAME individuals remain underrepresented in research, due to lack of inclusion by researchers, reluctance of participants and socioeconomic factors. ${ }^{34}$ Steps have been taken to address this: the UK's National Institute for Health Research initiated the Innovations in Clinical Trial Design and Delivery for under-served groups (INCLUDE) project to focus on making research more representative, and toolkits have also helped researchers to use appropriate language to engage with these groups..$^{35}$

\section{Racism is old news}

There is a long history of Black people being the unlawful subjects of unethical medical trials and testing. Dr ] Marion Sims carried out numerous experimental operations, without anaesthetic, on Black slave women between 1845 and $1849 .{ }^{36}$ In 1932, the Tuskegee Study of untreated syphilis in the 'negro male' enticed subjects under the guise of free health checks, meals and treatment. They were not told that they had syphilis and continued to receive a placebo even after the discovery of penicillin as an effective treatment to allow the evolution of the pathology to be understood through the exploitation of the study subjects. ${ }^{37}$

In more recent times, those with sickle cell disease, which has predominance within African and Caribbean communities, have been one the most marginalised and stigmatised groups. ${ }^{38}$ In the UK there are 15,000 people living with sickle cell disease making it one of the most common inherited, genetic conditions in the UK. ${ }^{39,40}$ Given its prevalence within the UK population and the health burden it places on patients there is still significant variation of care and awareness. These concerns were recognised and addressed by The Sickle Cell Society, with the introduction of Standards for the clinical care for adults with sickle cell disease in the UK, ${ }^{41}$ which focuses on the minimum standard of care patients should receive and outlines ways in which these standards can be achieved, the first of its kind. The report took 3 years to produce, with no funding from the NHS. The NHS has a responsibility and a duty of care to all; a great measure of the altruism of an institution is how it serves its most marginalised and vulnerable communities and this should not be left to small charities alone.

\section{Don't BAME me}

We have seen huge popularity of the acronym BAME, referring to 'Black, Asian and minority ethnic' communities. ${ }^{42}$ But how effective is this term in describing the myriad hyphenated identities within an ethnic minority population? More than 7.6 million people in the UK identified as 'BAME' in the 2011 census. ${ }^{43}$ But this group is not a monolith and the term does little to reflect the differences between ethnic minority subgroups.

The term has continued to be at the forefront of our vocabulary during the coronavirus pandemic, with 'BAME healthcare workers' being described as having the highest risk - but if you look more closely, data from the Office of National statistics showed that Black Africans were initially at highest risk within the BAME group..$^{44}$ This is not immediately clear without an astute attention to detail. This is in part because the term BAME is misleading. The letter ' $A$ ' means Asian, which, in itself, is a very broad term. Does it mean 'South Asian', 'East Asian', 'South East Asian', 'Indian', 'Pakistani', 'Chinese', 'Thai', 'Vietnamese'? The term classes all people with widely varying characteristics, genetics and cultures together, simply on the basis that they are not White. This may be in part related to the small size of subgroup but inappropriate aggregation of minority groups comes at the cost of true subgroup homogeneity, and masks the fact that severe health disparities often exist among racial and ethnic subgroups. In relation to the COVID crisis, breaking down at-risk groups by specific ethnicities would have allowed us to be best placed to understand the needs of individual communities.

Placing all ethnic minorities under the umbrella term BAME is reductive and can serve to entrench 'Whiteness' as normative while otherising and homogenising all other non-White identities. For example, the experience of Black and Asian people is different, especially within other areas of health. Maternal health has been seen as an area in which improvements can be made, given the disparity of outcomes for mothers of different ethnic backgrounds. The recent MBRRACE study looked at maternal death and morbidity for women in the UK and Ireland. Although outcomes were generally poorer for women from 'BAME' communities, drilling down to each ethnic group showed that Black women were more than five times as likely to die in child birth and Asian women twice as likely to die when compared to White women..$^{45}$ Although both are shocking statistics, it is clear there is a difference in health outcomes even within groups labelled BAME.

There are further limitations of this term when applied to the growing group of people with mixed heritage. Furthermore, the term BAME can be utilised to intentionally mislead. A local CCG 
produced the report Havering Clinical Commissioning Group Workforce Race Equality Standard 2018-2019.46 It found that 33\% of the workforce were BAME. The report stated, 'The CCG workforce has an overrepresentation of BME staff at 33\% when compared to the borough population of $12 \%$ (2011 Census). An underrepresentation of White staff (53\%) is evident, with the White population at $88 \%$.' After a telephone enquiry, it was discovered that none of the $33 \%$ described as BAME were Black. This specific information was not mentioned in the report. If broken down by ethnicity, would this still be considered an 'overrepresentation'? Many large organisations like the NHS may appear more diverse than they actually are based on the BAME term.

We must be intentional with being diverse and inclusive. Research suggests that mandatory policies are far more effective at achieving a diverse workforce that adequately provides for the communities they serve. ${ }^{7,47}$ An analysis into the affirmative action included an in-depth comparative case study exploring the practical applications of affirmative action measures in 11 countries including the UK, US, France and Netherlands. One of the concluding findings from the report was that

there was an overwhelming consensus that positive action is an effective and legitimate strategy to address past issues of inequality and unequal access for certain groups and for bringing about changes in private and governmental organisations, the health sector, educational institutions, and voluntary sector organisations.

This is a sentiment that could help address some the issues faced within our healthcare system. ${ }^{48}$

\section{Racism: a 'serious threat to public health'}

There is a catalogue of micro, macro and systematic-level aggressions that are experienced by ethnic minorities within the UK and these can often seem subtle to those with privilege who have not been subject to disadvantage as a visible person of colour. To dismantle racism, we must act together and formulate strategies to protect us all. In November 2020 The American Medical Association (AMA) took the step of declaring racism 'a serious threat to public health'. ${ }^{49}$ This is a huge step towards ensuring equality within healthcare. Adopting a systematic approach, the AMA appear dedicated to dismantling racism and the policies that impact access to healthcare. In order to tackle this 'disease', we must be actively anti-racist.

We must speak about the positive movements taking place within the NHS that are making great headway to improve representation. One striking area for improvement was in dermatology. Mind the gap, created by Malone Mukwende, a medical student from London, is a handbook of clinical signs in Black and Brown skin that aims to teach healthcare professionals about the importance of recognising how some conditions can present differently on darker skins. ${ }^{50}$ This is ground-breaking, not only because it demonstrates the importance of inclusivity in healthcare but also because it serves as a reminder of the importance of adequate training for healthcare professionals.

The RCGP has formed a taskforce comprised of diverse members working on aspects of representation, professional development and training. The RCGP has also responded to the requests made by its members to remove the term 'BAME' and are receiving advice on this. Along with other royal colleges, they are continuing work to address differential attainment. ${ }^{51}$ In July 2020 the Royal College of Obstetricians and Gynaecologists (RCOG) launched the Race Equality taskforce. Together with organisations like Five $X$ More, they work towards eradicating maternal health disparities in the UK. ${ }^{52}$ We have seen the effects of bias and unchallenged beliefs. Training is required for those working in the NHS, in particularly those in recruitment. We must try and rebuild trust within communities in a bid to improve access. Education is key to challenging thinking and practices for healthcare workers and service users alike.

However, change and policy movements do not have to start and end with one or two professional bodies. We all have a part to play to advocate for change which can be done in a number of ways, including but not limited to the following:6,53

> Governments and public agencies should seek to understand why inequalities exist and how racism and structural discrimination contribute to ethnic disparities in health with full inclusion of minority groups within this research.

> Health disparities should be highlighted and communicated to the general public, healthcare providers, healthcare professionals and other relevant bodies.

$>$ We should recognise the importance of representation, abolish barriers to progression and increase the proportion of ethnic minorities among medical workers and in leadership posts at all levels.

> Patient services can improve access to and experiences and outcomes of health and social care by reducing variations around best practices and promoting care through evidencebased guidelines.

$>$ We need adequate allocation of resources and funding to fulfil the above requirements.

Through this article we have illustrated examples of the disproportionate impact of racism on morbidity and mortality experienced by minority groups. We need a drive and commitment to change. This will enable us to better serve and meet the needs of the increasingly diverse population of the UK.

The conversations have commenced, we are seeing increasing accountability and most importantly the journey for equality and equitable healthcare for all is underway.

\section{References}

1 Szczepura A. Access to health care for ethnic minority populations. Postgrad Med J 2005;81:141-7.

2 World Health Organization. The United Kingdom: WHO Coronavirus Disease (COVID-19) Dashboard. https://covid19.who.int/region/ euro/country/gb.

3 British Medical Association. COVID-19: The risk to BAME doctors. BMA, 2021. Available from www.bma.org.uk/advice-and-support/ covid-19/your-health/covid-19-the-risk-to-bame-doctors.

4 Martineau A, Jolliffe D, Hooper R et al. Vitamin D supplementation to prevent acute respiratory tract infections: systematic review and meta-analysis of individual participant data. BMJ 2017;356:i6583.

5 Tillin T, Forouhi N, McKeigue P et al. Cohort profile of SABRE, a UK population-based comparison of cardiovascular disease and diabetes in people of European, Indian Asian and African Caribbean origins. Int J Epidemiol 2010;41:33-42.

6 Public Health England. Beyond the data: Understanding the impact of COVID-19 on BAME groups. PHE, 2020. Available from www.gov. uk/government/publications/covid-19-understanding-the-impacton-bame-communities. 
7 House of Commons House of Lords Joint Committee on Human Rights. Black people, racism and human rights: Eleventh Report of Session 2019-21. UK Parliament, 2020. Available from https:// committees.parliament.uk/work/409/Black-people-racism-andhuman-rights/publications/.

8 The WRES Implementation team. NHS workforce Race Equality Standard 2019 data analysis report for NHS trusts. NHS England 2020. Available from www.england.nhs.uk/publication/workforce-race-equality-standard-data-reporting-2019/.

9 Francis R. Freedom to speak up - an independent review into creating an open and honest reporting culture in the NHS. Freedom to speak up, 2015. http://freedomtospeakup.org.uk/the-report/.

10 Torchia M, Calabrò A, Huse M. Women directors on corporate boards: from tokenism to critical mass. J Business Ethics 2011;102: 299-317.

11 Kar P. To tackle racism, the NHS needs policies with teeth. BMJ 2020;369:m2583.

12 Esmail A, Roberts C. Academic performance of ethnic minority candidates and discrimination in the MRCGP examinations between 2010 and 2012: analysis of data. BMJ 2013;347:f5662.

13 Esmail A, Everington S. The perils of researching racial discrimination. BMJ Opinion, 2020. https://blogs.bmj.com/bmj/2020/02/13/ aneez-esmail-and-sam-everington-the-perils-of-researching-racialdiscrimination [Accessed 29 November 2020].

14 Rimmer A. RCGP will work with international doctors on membership exam issues. BMJ. 2014;348:g4250.

15 Woolf K, Potts H, McManus I. Ethnicity and academic performance in UK trained doctors and medical students: systematic review and meta-analysis. BMJ 2011;342:d901.

16 The Department of Health and Social Care. The NHS Constitution for England. DHSC, 2015. www.gov.uk/government/publications/ the-nhs-constitution-for-england.

17 Du Bois W. The conservation of races. American Negro Academy, 1897.

18 Witherspoon D, Wooding S, Rogers A et al. Genetic similarities within and between human populations. Genetics 2007;176:351-9.

19 Hoffman K, Trawalter S, Axt J, Oliver M. Racial bias in pain assessment and treatment recommendations, and false beliefs about biological differences between Blacks and Whites. Proc Natl Acad Sci USA 2016;113:4296-301.

20 Goyal M, Kuppermann N, Cleary S, Teach S, Chamberlain J. Racial disparities in pain management of children with appendicitis in emergency departments. JAMA Paediatrics 2015;169:996.

21 Todd K, Deaton C, D'Adamo A, Goe L. Ethnicity and analgesic practice. Ann Emerg Med 2000;35:11-6.

22 Hall W, Chapman M, Lee K et al. Implicit racial/ethnic bias among health care professionals and its influence on health care outcomes: a systematic review. Am J Public Health 2015;105: e60-76.

23 Greenwood B, Hardeman R, Huang L, Sojourner A. Physicianpatient racial concordance and disparities in birthing mortality for newborns. Proc Natl Acad Sci USA 2020;117:21194-200.

24 Lane D. Ethnic differences in hypertension and blood pressure control in the UK. QJM 2001;94:391-6.

25 National Institute of Healthcare Excellence. Hypertension in adults: diagnosis and management. NICE guideline [NG136]. NICE, 2019. www.nice.org.uk/guidance/ng136.

26 National Statistics. Health Survey for England - 2004, Health of Ethnic Minorities, Main report. NHS Digital, 2006. Available from https://digital.nhs.uk/data-and-information/publications/statistical/ health-survey-for-england/health-survey-for-england-2004-healthof-ethnic-minorities-main-report.

27 Leenen F, Nwachuku C, Black H et al. Clinical events in high-risk hypertensive patients randomly assigned to calcium channel blocker versus angiotensin-converting enzyme inhibitor in the antihypertensive and lipid-lowering treatment to prevent heart attack trial. Hypertension 2006:48:374-84.
28 Vyas D, Eisenstein L, Jones D. Hidden in plain sight — reconsidering the use of race correction in clinical algorithms. New Engl J Med 2020;383:874-82

29 Dimsdale J. Psychological stress and cardiovascular disease. J Am Coll Cardiol 2008;51:1237-46.

30 Brondolo E, Love E, Pencille M, Schoenthaler A, Ogedegbe G. Racism and hypertension: a review of the empirical evidence and implications for clinical practice. Am J Hypertension 2011;24:518-29.

31 Salway S, Holman D, Lee $C$ et al. Transforming the health system for the UK's multiethnic population. BMJ 2020;368:m268.

32 Dhairyawan R. Evaluating values. BM] Leader, 2020. https://blogs. bmj.com/bmjleader/2020/06/15/evaluating-values-by-rageshridhairyawan/.

33 Srinivasan S, Moser R, Willis G et al. Small is essential: importance of subpopulation research in cancer control. Am J Public Health 2015:105:S371-3.

34 Redwood S, Gill P. Under-representation of minority ethnic groups in research — call for action. B J Gen Pract 2013;63:342-3.

35 National Institute for Health Research. Improving inclusion of under-served groups in clinical research: Guidance from the NIHR INCLUDE project. NIHR, 2020. Available from www.nihr.ac.uk/ documents/improving-inclusion-of-under-served-groups-in-clinical-research-guidance-from-include-project/25435 [Accessed 30 November 2020].

36 Wall L. The medical ethics of Dr J Marion Sims: a fresh look at the historical record. J Medical Ethics 2006;32:346-50.

37 Reverby S. Tuskegee: could it happen again? Postgrad Med J 2001;77:553-4.

38 Bediako S, Lanzkron S, Diener-West M et al. The measure of sickle cell stigma: initial findings from the improving patient outcomes through respect and trust study. J Health Psychol 2014;21:808-20.

39 Sickle Cell Society. About sickle cell. www.sicklecellsociety.org/ about-sickle-cell/

40 Dormandy E, James J, Inusa B, Rees D. How many people have sickle cell disease in the UK? J Public Health 2018;40:e291-5.

41 Sickle Cell Society. Standards for clinical care of adults with sickle cell disease in the UK. Sickle Cell Society, 2018. Available from www.sicklecellsociety.org/sicklecellstandards/.

42 Aspinall P. BAME (Black, Asian and minority ethnic): the "new normal' in collective terminology. J Epidemiol Community Health 2021;75:107.

43 Office of National Statistics. 2011 Census analysis: Ethnicity and religion of the non-UK born population in England and Wales: 2011. ONS, 2020. Available from: www.ons.gov.uk/peoplepopulationandcommunity/culturalidentity/ethnicity/articles/ 2011 censusanalysisethnicityandreligionofthenonukbornpopulat ioninenglandandwales/2015-06-18

44 Office of National Statistics. Updating ethnic contrasts in deaths involving coronavirus COVID-19: England and Wales, deaths occurring 2 March to 28 July 2020. ONS, 2020. Available from www. ons.gov.uk/releases/explainingethnicbackgroundcontrastsindeath sinvolvingcovid19england2ndmarchto3rdjuly2020.

45 Knight M. MBRRACE-UK update: Key messages from the UK and Ireland Confidential Enquiries into Maternal Death and Morbidity 2019. Obstet Gynaecol 2020;22:93-5.

46 Havering Clinical Commissioning Group. Havering Clinical Commissioning Group Workforce Race Equality Standard 2018-2019. Havering Clinical Commissioning Group, 2020. www. haveringccg.nhs.uk/downloads/BHR-CCGs/News-and-pub/Strategicdocuments/NHS-Havering-CCG-Workforce-race-equality-standard2018-19-final.pdf.

47 Kalev A, Dobbin F, Kelly E. Best practices or best guesses? Assessing the efficacy of corporate affirmative action and diversity policies. Am Soc Rev 2006;71:589-617.

48 Archibong U, Sharps P. A comparative analysis of affirmative action in the United Kingdom and United States. J Psychol Issues Org Culture 2013;3(S1):28-49. 
49 American Medical Association. New AMA policies recognize race as a social, not biological, construct. AMA, 2020. www.ama-assn.org/ press-center/press-releases/new-ama-policies-recognize-race-socialnot-biological-construct.

50 Mukwende M, Tamony P, Turner M. Mind the Gap: A handbook of clinical signs in Black and brown skin. www.blackandbrownskin. co.uk/mindthegap.

51 Howe A, Marshall M, Vaughan-Dick V. Better for us all — recent learning on how the Royal College of General Practitioners can reduce racism. BJGP Open 2020; 4:bjgpopen20X101150.

52 Royal College of Obstetricians and Gynaecologists. Race Equality Taskforce. www.rcog.org.uk/en/news/campaigns-and-opinions/ race-equality-taskforce/.
53 Smedley B, Stith A, Nelson A (eds). Unequal treatment: confronting racial and ethnic disparities in health care. National Academies Press, 2003.

Address for correspondence: Dr Y Danso, Fairfield House, Fairfield General Hospital, Rochdale Old Road, Bury BL9 7TD, UK.

Email:yaadanso@doctors.org.uk

Twitter: @clinicdiaries

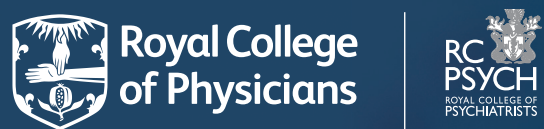

\section{Support your peers during the COVID-19 pandemic}

Peer support can help staff cope with the stress that comes from their work. New guidance from the Royal College of Physicians and Royal College of Psychiatrists sets out the values, principles and practice of peer support.

Download the guidance and accompanying infographic: www.rcplondon.ac.uk/projects/peer-support-guidance
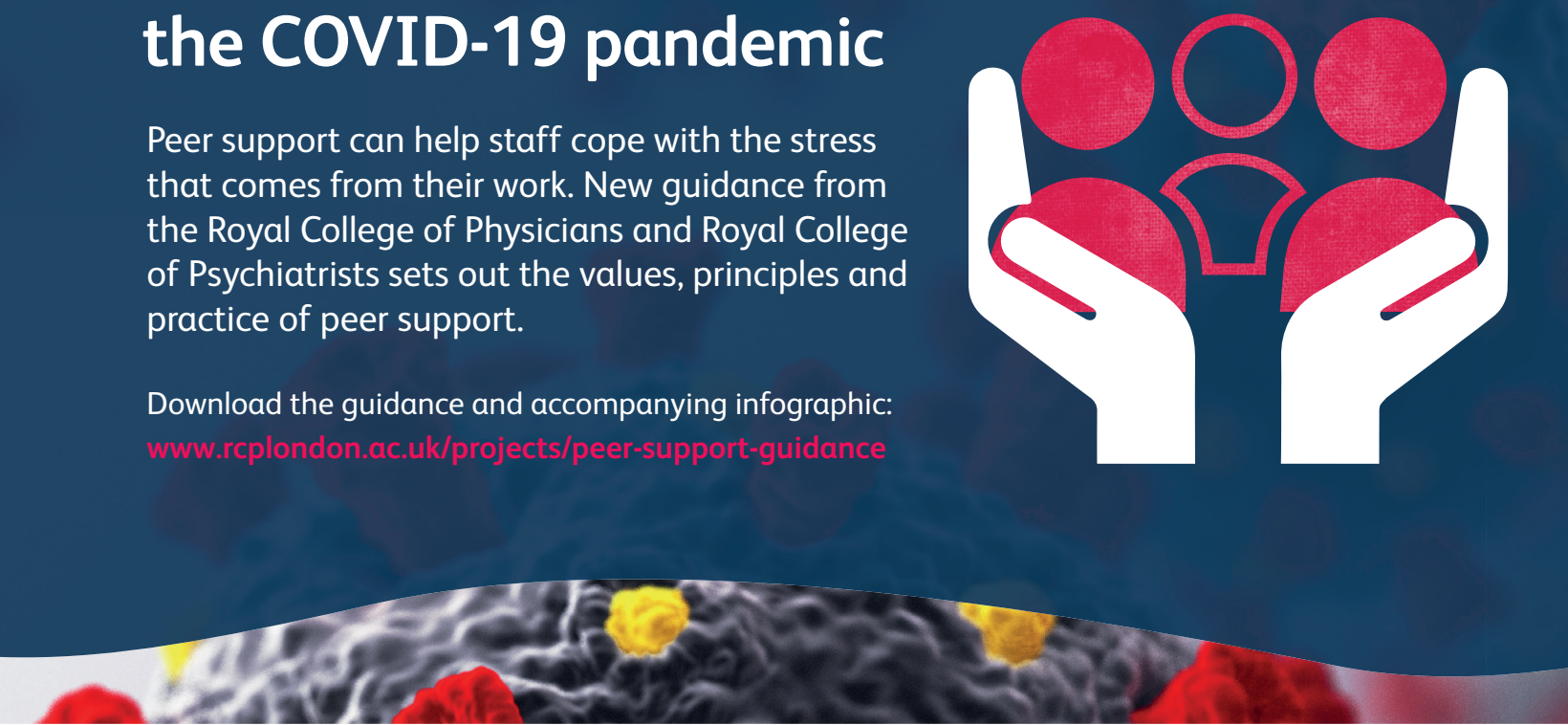